\title{
Convolution Theorem for Distributional Fourier-Stieltjes Transform
}

\author{
B. G. Khedkar ${ }^{1}$, S. B. Gaikwad ${ }^{2}$ \\ ${ }^{1}$ Arts, Commerce and Science College Sonai, Newasa, Ahmednagar, India \\ ${ }^{2}$ New Arts, Commerce and Science College Ahmednagar, India
}

*Corresponding Author: B. G. Khedkar, Arts, Commerce and Science College Sonai, Newasa, Ahmednagar, India

\begin{abstract}
In the tremendous expanding knowledge of science, mathematics plays vital role. In the words of Philip, Mathematics is a science of quantity and space. Especially in quantam field theory, field of partial differential equations, Harmonic analysis etc.the notion of generalized functions is very essential.The convolution theorem of the transform plays an important role in digital signal processing.The usefulness of convolution theorem can be best explained by its applications in filtering. This paper is concerned with the generalization of Fourier-Stieltjes transform in the distributional sense. The main aim of this paper is to prove properties of convolution and convolution theorem for Fourier-Stieltjes transform.
\end{abstract}

Keywords: Fourier transform, Stieltjes transform, Fourier-Sieltjes transform, Integral transform, Convolution.

AMS Mathematics Subject Classification: (2010) 46F12

\section{INTRODUCTION}

In mathematics and, in particular, functional analysis, convolution is a mathematical operation on two functions $\mathrm{f}$ and $\mathrm{g}$, providing a third function that is typically viewed as a modified version of one of the original functions, giving the area overlap between the two functions as a function of the amount that one of the original functions translated. Convolution is similar to cross-correlation. It has applications that include probability, statistics, computer vision, natural language processing, image and signal processing electrical engineering and differentials equations. Generalizations of convolution have applications in field of numerical analysis, numerical linear algebra and in the design and implementation of finite implse response filters in signal processing.

The integral transforms plays an important role in signal processing. Fourier analysis is one of the frequently used tools in signal processing and many other scientific disciplines. The Stieltjes transform has been widely used in applied mathematics. Here we discuss the properties of convolution and convolution theorem for Fourier-Stieltjes transform which is very applicable. The convential Fourier-Stieltjes transform of complex valued smooth function $\mathrm{f}(\mathrm{t}, \mathrm{x})$ is defined by the convergent integral.

$$
\mathrm{F}(\mathrm{s}, \mathrm{y})=\mathrm{FS}\{\mathrm{f}(\mathrm{t}, \mathrm{x})\}=\int_{0}^{\infty} \int_{0}^{\infty} f(t, x) e^{-i s t}(x+y)^{-p} d t d x
$$

\section{DEFINITIONS}

\subsection{The function Space:The Space $\mathrm{FS}_{\boldsymbol{\alpha}}$}

A function $\mathrm{f}$ defined on $0<\mathrm{t}<\infty, 0<\mathrm{x}<\infty$ is said to be member of $\mathrm{FS}_{\alpha}$ if $\phi(\mathrm{t}, \mathrm{x})$ is smooth for each non-negative integer 1 , q.

$$
\begin{gathered}
\gamma_{k, p, l, q} \phi(t, x)=\sup _{I}\left|t^{k}(1+x)^{p} D_{t}^{l}\left(x D_{x}\right)^{q} \phi(t, x)\right| \\
\leq C_{l q} A^{p} \cdot p^{p} \quad \mathrm{p}=1,2,3,--------
\end{gathered}
$$


Where the constant $\mathrm{A}$ and $\mathrm{C}_{1 \mathrm{q}}$ depend on the testing function $\phi$.

The space FS $\alpha$ areequiparallel with their natural Housdoff locally topology $\tau_{\alpha}$. This topology is respectively generated by the total families of semi norms $\left\{\gamma_{\mathrm{k}, \mathrm{p}, 1, \mathrm{q}}\right\}$ given by (2.1).

\subsection{Distributional Fourier-Stieltjes transform of generalized function in $F S_{\alpha}^{*}$}

Let $F S_{\alpha}^{*}$ is the dual space $\mathrm{FS}_{\alpha}$. This space $F S_{\alpha}^{*}$ consists of continuous linear function on FS ${ }_{\alpha}$.

Let $\phi(t, x) \in F S_{\alpha}^{*}$, for some $\mathrm{s}>0$ and $\mathrm{k}>\mathrm{Re} \mathrm{p}$, then distributional Fourier-Stieltjes Transform $\mathrm{F}(\mathrm{s}, \mathrm{y})$ of

$$
\mathrm{FS}\{\mathrm{f}(\mathrm{t}, \mathrm{x})\}=\mathrm{F}(\mathrm{s}, \mathrm{y})=\left\langle f(t, x), e^{-i s t}(x+y)^{-p}\right\rangle
$$

Where for each fixed $\mathrm{t}(0<\mathrm{t}<\infty), \mathrm{x}(0<\mathrm{x}<\infty)$ the right side of above equation has same as an application of $f(t, x) \in F S_{\alpha}^{*}$ to $e^{-i s t}(x+y)^{-p} \in F S_{\alpha}$.

\section{3. Fourier-Stieltjes Type Convolution:}

Fourier-Stieltjes type convolution is an operation that assigns to each arbitrary pair $f \in F S_{\alpha}^{*}$ and $g \in F S_{\alpha}^{*}$, the Fourier-Stieltjes type convolution $f * g \in F S_{\alpha}^{*}$ defined by

$$
\langle f * g, \phi\rangle=\langle f(t, x),\langle g(s, y), \phi(t+s, x+y)\rangle\rangle, \text { where } \phi \in F S_{\alpha}
$$

\section{Main Results}

\subsection{Theorem:}

If $f(t, x) \in F S_{\alpha}^{*}$ and $\phi(t, x) \in F S_{\alpha}$ then $\phi \rightarrow \psi$ is a continuous linear mapping of $F S_{\alpha} \rightarrow F S_{\alpha}$, where $\psi(s, y)=\langle f(t, x), \phi(t+s, x+y)\rangle$

Proof: By induction method we can show that

$$
D_{s, y}^{m+n} \psi(s, y)=\left\langle f(t, x), D_{s, y}^{m+n} \phi(t+s, x+y)\right\rangle
$$

For showing $\psi(t, x) \in F S_{\alpha}$, consider

$$
\begin{aligned}
& =\sup _{I}\left|s^{k}(1+y)^{p} D_{s}^{l}\left(y D_{y}\right)^{q}\langle f(t, x), \phi(t+s, x+y)\rangle\right| \\
& \leq C \max _{\substack{0 \leq n \leq r_{2} \\
0 \leq m \leq n}} \sup _{I}\left|s^{k}(1+y)^{p} D_{s}^{l}\left(y D_{y}\right)^{q} t^{k}(1+x)^{q} D_{s}^{m}\left(x D_{x}\right)^{q} \phi(t+s, x+y)\right|
\end{aligned}
$$

Where $\mathrm{r}_{1}$ and $\mathrm{r}_{2}$ are non-negative integers depending on $\mathrm{f}$.

$$
\begin{aligned}
& \leq C \max _{\substack{0 \leq n \leq r_{2} \\
0 \leq m \leq n}} \sup _{I}\left|s^{k}(1+y)^{p} t^{k}(1+x)^{q} D_{s, y}^{l+q} D_{s, y}^{m+n} \phi(t+s, x+y)\right| \\
& \leq C \max _{\substack{0 \leq n \leq r_{2} \\
0 \leq m \leq n}} \gamma_{k, p, l+m, q+n}(\phi) \quad(3.1 .2)
\end{aligned}
$$

Thus $\psi \in F S_{\alpha}$ continuity follows from (3.1.2) and hence theorem. $\square$

\section{Properties of Fourier-Stieltjes Type Convolution}

\subsection{Theorem:}

If $f(t, x) \in F S_{\alpha}^{*}$ and $g \in D_{+}(I)$ then $g \rightarrow f * g$ is continuous linear map from $\mathrm{D}_{+}$into $\mathrm{E}_{+}$, where $(f * g)(s, y)=\langle f(t, x), g(s-t, y-x)\rangle$. sagggd

Proof: It is easy to prove that $f * g$ ) is smooth and the mapping is linear. 
For its continuity,

$$
\begin{aligned}
& \left|D_{s, y}^{k_{1}+k_{2}}(f * g)(s, y)\right|=\left|\left\langle f(t, x), D_{s, y}^{k_{1}+k_{2}}\{g(s-t, y-x)\}\right\rangle\right| \\
& \quad \leq C \max _{\substack{0 \leq q+k_{2} \leq r_{2} \\
0 \leq l+k_{1} \leq r_{n}}} \sup _{I}\left|D_{l, x}^{l+q} D_{s, y}^{k_{1}+k_{2}}\{g(s-t, y-x)\}\right|
\end{aligned}
$$

Since $g \in D_{+}(I)$, continuity follows from above inequality. We call $(f * g)(s, y)=\langle f(t, x), g(s-t, y-x)\rangle$ as Fourier-Stieltjes type regularization. $\square$

\subsection{Theorem:}

Convolution operation in (3.2) commutes with shifting scaling operator S i.e. $S(f * g)=f *(S(g))$

Proof: Consider

$$
\begin{aligned}
& \langle S(f * g), \phi(t, x)\rangle=\langle f * g, \phi(t+s, x+y)\rangle \\
& =\langle f,\langle g, \phi(s-t, y-x)\rangle\rangle
\end{aligned}
$$

Now $\langle f * S(g), \phi(t, x)\rangle=\langle f,\langle S(g), \phi(t+s, x+y)\rangle\rangle$

$$
=\langle f,\langle g, \phi(s-t, y-x)\rangle\rangle
$$

Therefore from (3.3.1) and (3.3.2), we write

$$
S(f * g)=f *(S(g))
$$

\subsection{Theorem:}

$f \in D_{+}^{*}$ and $F S\{(u, v)\}=f(s, y)$, and $y \in \Omega_{f}$ and $g \in D_{+}^{*}, F S\{g(t, x)\}=G(s, y)$, s and $\mathrm{y} \in \Omega_{g}$ and $\Omega_{f} \cap \Omega_{g}$ is not empty, then $f * g$ exists in the sense of FS-type convolution in $F S_{\alpha}^{*}$ where the strip of definition is the intersection of $\Omega_{f} \cap \Omega_{g}$ with real axis. Moreover $F S(f * g)=F S(f) . F S(g)$

Proof: Using theorem (3.3) it can be easily shown that $f * g \in F S_{\alpha}^{*}$.

Further as $K(t, x, s, y)=e^{-i s t}(x+y)^{-p} \in F S_{\alpha}$ for each fixed s and $\mathrm{y}$

$$
\begin{aligned}
& F S(f * g)=\left\langle f * g, e^{-i s t}(x+y)^{-p}\right\rangle \\
& =\left\langle f(u, v),\left\langle g(t, x), e^{-i s(t+u)}(v+y)^{-p}(x+y)^{-p}\right\rangle\right\rangle \\
& =\left\langle f(u, v), e^{-i s u}(v+y)^{-p}\right\rangle\left\langle g(t, x), e^{-i s t}(x+y)^{-p}\right\rangle \\
& =F S\{f(u, v\} . F S\{g(t, x)\} \\
& =F(s, y) . G(s, y)
\end{aligned}
$$

Hence the theorem.

\section{CONCLUSIONS}

This paper is concerned with the generalization of Fourier-Stieltjes transform in the distributional sense. In this paper the Fourier-Stieltjes type convolution and its properties are proved.

\section{ACKNOWLEDGMENTS}

We would like to express our deep sense of gratitude to authors and scientists, whose work provides a valuable source of inspiration in undertaking the present work. 


\section{REFERENCES}

[1] Sharma V.D. and Rangari A. N., Convolution Theorem for Distributional Fourier-Laplace Transform, International Journal of Research in Engineering and Applied Sciences, Vol.5,Issue 12,pp.142148,Dec.2015.

[2] Sharma V.D. and DolasP.D.,Analyticity of Distribution Generalized Fourier-Stieltjes Transforms,International Journal of Mathematical Analysis,Vol.6,No.9,pp.447-451,2012.

[3] ZemanianA.H.,Generalized integral transform, Inter science publisher,New York, 1968.

[4] ZemanianA.H.,Distribution theory and transform analysis, McGraw Hill,New York, 1965.

[5] Lighthill M.J.; \{Fourier analysis and generalized functions $\}$ lemph\{Cambridge University Press \}, (1960).

[6] Gaikwad S.B., Generalized Fourier-Modified Stieltjes Transforms,Proceedings of Role of Mathematics in Science Engineering and Techonology,2015

[7] Pathak R.S.,A Course in Distribution Theory and Applications,CRC Press 2001.

[8] PathakR.S.,Integral Transformation of Generalized Functions and Their Applications,Hordan and Breach Science Publications, Netherland.

[9] Gupta Anupama,Fourier Transform and Its Applications in Cell Phones, International Journal of Scientific and Research Publications, Volume 3,Issue 1,PP.1-2,January 2013.

\section{AUTHORS' BIOGRAPHY}

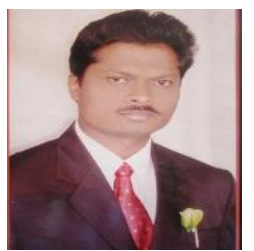

Mr. KhedkarBalasaheb G. currently works as an Assistant Professor at Arts, Commerce and Science College Sonai, Ahmednagar, Maharashtra, India. He also serves as the Head of Mathematics Department there. Mr. Khedkar completed his M.Sc. degree from Pune University and M. Phil from Madurai Kamaraj University. His area of interest is integral transforms on generalized functions.

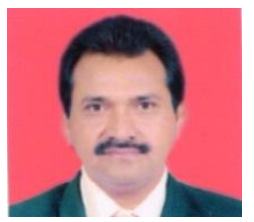

Dr. Gaikwad Shrikisan Baburao currently works as an Associate Professor, Head P.G. Department and Research Centre at New Arts, Commerce and Science Ahmednagar, Maharashtra, India. He has completed his M.Sc. degree from Shivaji University Kolhapur and also M. Phil and $\mathrm{Ph}$. D from the same University. He serves as a research guide for M. Phil and Ph.D. under SavitribaiPhule Pune University. Two students have completed their Ph.D. under his guidance His area of interest is integral transforms on generalized functions.

Citation: B. G. Khedkar, S. B. Gaikwad, "Convolution Theorem for Distributional Fourier-Stieltjes Transform ", International Journal of Scientific and Innovative Mathematical Research, vol. 5, no. 9, p. 3942, 2017., http://dx.doi.org/10.20431/2347-3142.0509004

Copyright: (C) 2017 Authors. This is an open-access article distributed under the terms of the Creative Commons Attribution License, which permits unrestricted use, distribution, and reproduction in any medium, provided the original author and source are credited. 